\title{
Eu quero, eu posso, mas será que eu consigo? Fenômeno Impostor em pós-graduandos stricto sensu da área de negócios
}

\author{
Alison Martins Meurer \\ https://orcid.org/0000-0002-3704-933X \\ Flaviano Costa \\ https://orcid.org/0000-0002-4694-618X
}

\section{Resumo}

Objetivo: Identificar os fatores que caracterizam o Fenômeno Impostor de pós-graduandos stricto sensu da área de negócios.

Método: a pesquisa contou com a participação válida de 613 discentes nas fases de pré-teste e 1.816 discentes na coleta final. Os dados foram analisados por meio de estatística descritiva, Análise Fatorial Confirmatória e Análise Fatorial Exploratória.

Resultados: os indicadores da CIPS apontaram para uma bidimensionalidade, sendo que um dos fatores caracteriza sentimentos de falsidade e subestimação e outro relacionado à sorte ou ao acaso. Os resultados ainda apontam que os sentimentos impostores na área de negócios estão mais voltados para a percepção de falsidade ou subestimação, do que para a atribuição do sucesso à sorte ou ao acaso.

Contribuições: a identificação do Fenômeno Impostor é importante para que esses sentimentos possam ser moldados a fim de não limitar e prejudicar o desenvolvimento acadêmico e a carreira profissional dos discentes. Palavras-chave: Fenômeno Impostor; Pós-graduação stricto sensu; Área de negócios. 


\section{Introdução}

Fenômeno Impostor (FI) é o termo utilizado para caracterizar indivíduos que atribuem seu sucesso à sorte ou a algum outro fator que não seja a sua própria capacidade física ou intelectual. Esse fenômeno desperta sentimentos de insegurança em indivíduos bem-sucedidos que os fazem crer que o sucesso somente é alcançado por meio da sorte ou de esforços grandiosos (Clance \& Imes, 1978; Cisco, 2020). A temática tem ganhado os holofotes após celebridades mundiais (Celebrity Insider, 2017; Idiva, 2019; Marie Claire, 2019) e executivos de grandes empresas (Entrepreneur, 2017) terem admitido publicamente que convivem com sentimentos impostores. Especialistas também vieram a público para alertar que as consequências do FI podem vir "sob a forma de estresse; ficar preso em um emprego por muito tempo; perder promoções, experiências valiosas de aprendizado ou conexões; e esgotamento. E em quase todos os casos, há um custo financeiro" (Young, 2019, n. p. publicado em The Enterprises Project, 2019).

$\mathrm{Na}$ pós-graduação, é comum haver a manifestação de sentimentos advindos do FI, visto que estes estudantes estão envoltos em um ambiente que requer o desenvolvimento de atividades criativas, competição, cumprimento de prazos e avaliação por parte de terceiros que delineiam um contexto favorável à manifestação de sentimentos impostores (Levecque et al., 2017; Cisco, 2020). Ainda em relação ao meio acadêmico, mesmo com a intensificação das demandas de investigações sobre FI, como pesquisas precursoras nos moldes da realizada por Thompson, Davis e Davidson (1998), ainda é incipiente e pouco explorado este fenômeno em estudos científicos. No stricto sensu, as evidências ainda são embrionárias e insuficientes para compreender o FI e suas consequências nesse nível de ensino (Craddock, Birnbaum, Rodriguez, Cobb, \& Zeeh, 2011).

$\mathrm{Na}$ área de negócios, formada por cursos de Administração, Contabilidade e Economia, as pesquisas são ainda mais escassas, sendo que há estudos que indicam peculiaridades do comportamento dos discentes destes cursos em relação aos demais (McCabe, Butterfield, \& Treviño, 2006). A propensão dos discentes da área de negócios para manifestar o FI se deve pela cobrança por competitividade e eficiência que permeia esse campo de atuação. Tais preocupações são relevantes à medida que os acadêmicos atuais serão os possíveis gestores, professores e líderes em um futuro próximo. Além disso, espera-se que o contexto universitário auxilie no desenvolvimento da responsabilidade e liderança dos discentes (Freire, 2014) tornando importante direcionar atenção para possíveis interventores dessa formação, neste caso o FI.

Ademais, o excesso de expectativas por parte de família, professores e instituição direcionadas aos estudantes do ensino superior podem maximizar os sentimentos impostores. Nos níveis de pós-graduação, esse problema mostra-se ainda maior, pois as diferentes experiências e formações existentes entre os discentes podem favorecer a assimilação de inadequação e o afloramento do FI (Craddock et al., 2011). Desse modo, o FI caracteriza-se como uma problemática importante a ser investigada a fim de, a partir da sua identificação, possibilitar o delineamento de ações que auxiliem os indivíduos a melhorarem seus níveis de bem-estar, assimilação da capacidade intelectual e de sucesso. As ações podem ser orientadas para o desempenho acadêmico e bem-estar dos pós-graduandos stricto sensu e aprimoramento do ambiente dos programas de pós-graduação. Portanto, o objetivo do estudo consiste em identificar os fatores que caracterizam o Fenômeno Impostor de pós-graduandos stricto sensu da área de negócios. 
Os achados da pesquisa indicam que um a cada cinco participantes possuem sentimentos impostores frequentes ou intensos, sendo que estes sentimentos se caracterizam pela subestimação de suas habilidades e competências ou atribuição de seu sucesso à sorte. Logo, a relevância do estudo é pautada no fato de que se espera que pós-graduandos stricto sensu ao final do curso tenham desenvolvido a capacidade de analisar e solucionar problemas complexos, gerenciar projetos, possuir pensamento crítico, capacidade de liderança e gerenciamento do tempo (Andrade, 2018), sendo que parte dessas habilidades está relacionada direta ou indiretamente aos seus níveis de confiança sobre suas competências e proatividade para realizar atitudes positivas no ambiente social, já que a confiança é afetada pelo FI. Assim, é oportuna a identificação e a compreensão desses sentimentos nos discentes.

A pesquisa também apresenta contribuições no campo metodológico, visto que é realizada a tradução e validação da escala CIPS (Clance, 1985), instrumento desenvolvido com o intuito de mensurar o FI, sendo uma contribuição direta para que estudos futuros possam analisar esse fenômeno. Esta pesquisa também contribui com a ampliação das investigações sobre Fenômeno Impostor no ambiente do stricto sensu.

\section{Fenômeno Impostor: da origem às consequências}

O termo Fenômeno Impostor originou-se dos estudos de Clance e Imes, que acompanharam por aproximadamente cinco anos mais de 150 mulheres consideradas bem-sucedidas, que atuavam em diversas áreas profissionais, com níveis de graduação e pós-graduação, mas que possuíam dúvidas, inseguranças e sentimento de culpa acerca do seu sucesso (Clance \& Imes, 1978; Taylor, 2009). As descobertas da pesquisa indicaram que entre as principais características dos impostores estão o medo que alguém com cargos hierárquicos superiores observe que os níveis de competência não são tão altos como se pensava e a dificuldade em internalizar o sucesso obtido, normalmente atribuído à sorte ou ao trabalho árduo (Clance \& O’Toole, 1987).

Inicialmente, Clance e Imes (1978) mapearam quatro sintomas frequentemente relatados pelos impostores: (i) ansiedade generalizada; (ii) falta de autoconfiança; (iii) depressão; e (iv) frustração frente à incapacidade de cumprir os padrões e realizações autodefinidas. Posteriormente, Clance e O'Toole (1987) revelaram a associação do FI com diferentes traços de personalidade e sentimentos, compilando as principais características presentes naqueles que apresentam fortes convicções de medos impostores, sendo: [1] Introversão; [2] Ansiedade generalizada; [3] Dificuldade em aceitar elogios; [4] Superestimação das habilidades dos outros e subestimação das suas; [5] Culpa pelo sucesso; [6] Definição inadequada de inteligência; [7] Medo da avaliação; [8] Medo de falhar; [9] Mensagens ambientais e familiares; e [10] Ciclo do Impostor.

Essas características indicam que pessoas introvertidas tendem a exteriorizar comportamentos divergentes dos sentimentos vivenciados internamente. Com isso, a personalidade não é identificada nas relações sociais de forma alinhada com sua percepção do eu (Chassangre \& Callahan, 2017). Essa dissonância caracteriza um dos principais elementos do Fenômeno Impostor: a percepção de farsa. Os introvertidos ainda apresentam baixa autoconfiança e autoestima e níveis mais elevados de ansiedade que favorecem o impostorismo (Clance, 1985). Nesse escopo, Kets de Vries (1989, p. 21-22, tradução nossa) descreve os impostores como pessoas incomumente sensíveis às experiências de rejeição; eles são excessivamente receosos de falha social e sofrem de persistentes necessidades de dependência. $\mathrm{O}$ que também deve ser observado são suas atitudes perfeccionistas em relação a si mesmos. É como se eles incorporassem as expectativas excessivas de seus pais, que nunca foram devidamente "metabolizadas". Em muitos casos, eles podem sofrer de formas generalizadas de ansiedade, falta de autoconfiança e depressão. 
A baixa autoestima dificulta a aceitação de elogios e a internalização dos sucessos obtidos, pois o reconhecimento de suas competências por terceiros é assimilado pelos impostores como "uma indicação de que eles conseguiram enganar os outros para acreditar que eles são bem-sucedidos" (Sonnak \& Towell, 2001, p. 864). A insegurança frente às suas capacidades intelectuais fomenta a superestimação das habilidades das outras pessoas, e subestimação das suas próprias competências, perpetuando a baixa autoestima e sentimentos de inferioridade (Holmes et al., 1993; Chassangre, 2014). Prova disto é que na pesquisa de Sonnak e Towell (2001) a autoestima apresentou-se atrelada ao FI, sendo estabelecida relação inversa, na qual o aumento do FI está associado à baixa autoestima.

Adicionalmente, apesar de não ser assimilado internamente, o reconhecimento do sucesso desempenha papel ambíguo na vida daqueles que têm sentimentos impostores. Mesmo recusando externamente atitudes que enaltecem suas competências, ao obterem reconhecimento e elogios essas pessoas acreditam que estão conseguindo criar uma imagem social e enganar os que estão à sua volta (Ferrari \& Thompson, 2006). Parte desse comportamento deriva da forma como é assimilado o conceito de inteligência por estas pessoas. Normalmente, a inteligência é percebida de modo distorcido, como uma entidade fixa baseada no cumprimento de metas, com vistas a provar sua capacidade intelectual perante os outros. Ao falharem, os que possuem FI tendem a se sentirem inadequados, sentem vergonha, ansiedade, menores níveis de bem-estar e baixa autoestima são percebidos e, por isso, evitam situações em que podem ser avaliados. Já para os que não apresentam impostorismo, a inteligência é compreendida de forma maleável, voltada para a aprendizagem e o desenvolvimento intelectual, em que as falhas são encaradas como algo normal, que fortalecem a resiliência e auxiliam o crescimento pessoal (Chassangre \& Callahan, 2017). Parte desse comportamento é originado do ambiente familiar permeado pela alta cobrança e expectativas por sucessos (Langford \& Clance, 1993).

A atenção voltada para a percepção social de sua imagem e não ao desenvolvimento das tarefas, prende os indivíduos com FI a um comportamento cíclico que se perpetua e reforça os sentimentos impostores (Clance, Dingman, Reviere, \& Stober, 1995). Esse padrão comportamental é denominado na literatura como "Ciclo do Impostor" e envolve as diversas características e ações manifestadas durante a vida dos impostores (Thompson et al., 1998). A Figura 1 apresenta as fases desse ciclo.

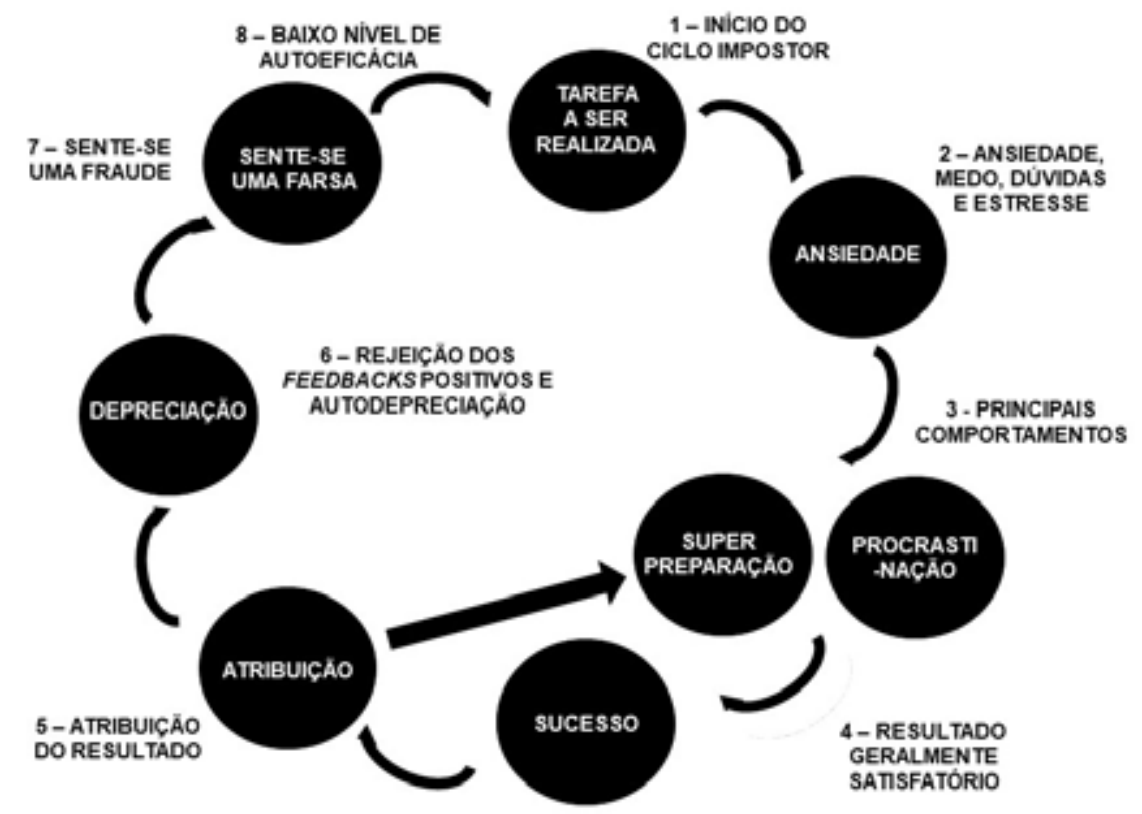

Figura 1. Ciclo do Impostor

Fonte: Chassangre e Callahan (2017, p. 102) adaptado de Clance (1985). 
A partir da Figura 1, percebe-se que, quando atribuída uma nova tarefa, os impostores enfrentam ansiedade (Chassangre \& Callahan, 2017), insegurança e medo (Clance, 1985) sobre sua capacidade de cumprir os desafios com sucesso. Esses indivíduos comumente reagem aos elevados níveis de ansiedade e insegurança de duas formas: a primeira é por meio da superpreparação, enquanto a segunda é caracterizada pela procrastinação seguida de elevada dedicação à tarefa (Thompson et al., 1998).

A superpreparação é caracterizada por comportamentos workaholics, que incluem horas de dedicação ao trabalho e/ou ao estudo em face da abdicação de outras atividades, a fim de superar sentimentos de ilegitimidade, evidenciando aspectos perfeccionistas. Por outro lado, a procrastinação consiste no baixo compromisso com o objetivo estabelecido e o desejo de proteger a autoestima por meio da protelação da tarefa (Chassangre \& Callahan, 2017). Quando próximo do limite para consecução da atividade, o indivíduo procrastinador se dedica intensamente para finalizar o que lhe foi solicitado. Desta maneira, a assimilação do sucesso tende a ser induzida pela postura adotada durante a execução da tarefa, seja de superpreparação ou procrastinação seguida de preparo intenso. Quando obtém sucesso na atividade, o indivíduo tende a não reconhecer sua conquista como algo legítimo (Thompson et al., 1998). Se sua postura foi de extrema preparação, então o sucesso é atribuído ao esforço elevado. De outro modo, se o comportamento foi de procrastinação, atribui-se a conquista à sorte ou a qualidades vinculadas à manipulação do ambiente (Thompson, Foreman, \& Martin, 2000). Com isso, ao ser elogiado tende a depreciar suas habilidades perante terceiros e rejeitar feedbacks positivos, pois não se sente merecedor do reconhecimento. Se o sucesso não é alcançado, então sentimentos de fraude são fomentados e atitudes de self-handicapping, ou seja, autossabotagem, podem ser adotadas para justificar os fracassos perante os outros.

Assim, de qualquer forma, no último estágio do ciclo, os impostores se sentem uma "farsa", não internalizam os resultados alcançados como fruto das suas competências e inteligência e temem ser desmascarados e taxados como alguém incompetente (Clance \& Imes, 1978; Chassangre, 2014). Desse modo, "à medida que novas situações de realização são encontradas, ansiedades e dúvidas desaparecem e o ciclo começa de novo" (Thompson et al., 1998, p. 382, tradução nossa) reforçando a crença de incapacidade de obter sucesso (Chassangre, 2014).

Clance e Imes (1978) citam que é lento minimizar sentimentos impostores, pois é preciso modificar a percepção do indivíduo sobre sua própria personalidade. Weiner (1972) explica que os padrões de desempenho e o número de sucessos e fracassos experimentados auxiliam a moldar a atribuição dos resultados alcançados. Nessa linha, Thompson et al. (1998) acreditam que a intensidade do FI diminui com a idade, pois repetidas experiências positivas vivenciadas ao longo dos anos possibilitam a assimilação do sucesso e minimizam os efeitos dos medos impostores, reforçando a crença de que o FI pode ser minimizado com o decorrer do tempo.

Referente aos estudos anteriores, Thompson et al. (1998) analisaram o processo atribucional dos impostores após situações de sucesso ou fracasso. O estudo foi operacionalizado por meio de testes de diferenças de grupos. Os resultados indicaram que não há diferenças no nível de FI entre os gêneros. Além disso, quanto maior a idade, menores são os sentimentos impostores. Além disso, os achados mostraram que os impostores generalizam suas falhas, atribuem seu sucesso a fatores externos e estão mais propícios a problemas relacionados a depressão. 
Craddock et al. (2011) buscaram compreender, a partir de entrevistas, a manifestação do Fenômeno Impostor nos doutorandos de um programa de pós-graduação localizado nos Estados Unidos da América. Os autores identificaram que, na infância, são incutidas expectativas cotidianas de alto desempenho e aversão à falha que promovem traços de personalidade perfeccionistas. A não aceitação do fracasso faz com que a busca por feedbacks positivos e o esquivamento das críticas sejam constantes. Com isso, as cobranças pessoais por sucesso acadêmico e desempenho elevado são internalizadas, passam a fazer parte da vida do aluno e ocasionam reflexos na fase adulta. Os relatos pontuam que questões raciais e de background familiar, como pertencer à primeira geração da família a cursar doutorado, fomentam sentimentos impostores. Ainda é apontada a necessidade de haver equilíbrio entre as obrigações acadêmicas e outros aspectos da vida social dos discentes, bem como suporte social para estes estudantes.

Sonnak e Towell (2001) indicam que o estilo de criação parental se associa ao Fenômeno Impostor, principalmente em relação à superproteção dos pais. A superproteção colabora para que os pais assumam as responsabilidades e os projetos dos filhos com o intuito de minimizar as chances de falhas. Ao serem superprotegidas as crianças tendem a consolidar elevados anseios por conquistas e sucessos, e passam a evitar situações de fracasso. O problema é que os fracassos são necessários para a formação construtiva do caráter e da resiliência do indivíduo, e esse comportamento é mantido durante a fase adulta. Com isso, o senso de habilidades é prejudicado e o sucesso passa a não ser internalizado de forma satisfatória, pois a criança reconhece que a consecução das tarefas não se deve às suas competências próprias, mas às interferências de terceiros (Want \& Kleitman, 2006).

French, Ullrich-French e Follman (2008) validaram a Clance Impostor Phenomenon Scale (CIPS) em estudantes de engenharia norte-americanos. $\mathrm{O}$ modelo com os indicadores mais adequado foi composto de duas dimensões, uma relacionada à falsidade e outro inerente à sorte. Esses achados podem ser indícios de que as mudanças culturais ocorridas na sociedade ao longo dos anos podem ter afetado a unidimensionalidade da CIPS delineando um constructo multidimensional.

Deffendall, Knutson e Sacks (2011) investigaram o perfil dos estudantes de primeira geração de cursos de graduação de uma universidade do Reino Unido. Os achados indicaram que acadêmicos da primeira geração possuem maiores taxas de evasão do que aqueles em que pelo menos um dos pais possuem formação de nível superior. Para os autores, os desafios vivenciados no ensino superior podem fazer com que os discentes se sintam uma "fraude", e não possuir aconselhamento familiar adequado para lidar com essas situações colabora para que este grupo tenha maiores taxas de evasão universitária e sentimentos impostores.

Tais estudos conduzem a um campo teórico-empírico em evolução, que destaca a relevância de mensurar o FI no contexto universitário. Em se tratando do cenário brasileiro, validar a CIPS possibilita o desenvolvimento de novas investigações inerentes a temáticas, bem como maior confiabilidade nas comparações entre pesquisas que venham a ser realizadas no ambiente nacional. 


\section{Metodologia}

A coleta de dados foi operacionalizada por meio da plataforma on-line Survey Monkey e coletados entre agosto e dezembro de 2018. A população do estudo consiste nos 15.971 pós-graduandos stricto sensu regularmente matriculados na área de negócios, composta pelos cursos de Administração, Contabilidade e Economia. A amostra contou com 1.816 participações válidas, sendo atendido o parâmetro mínimo amostral de 375 participantes ao nível de significância de 95\% e margem de erro de 5\% (Survey Monkey, 2021). Os pré-testes foram realizados com pós-graduandos stricto sensu de outras áreas do conhecimento, a fim de verificar a confiabilidade do instrumento de coleta de dados e não enviesar os respondentes da amostra da pesquisa. O Fenômeno Impostor foi mensurado por meio da Clance Impostor Phenomenon Scale que originalmente é composta por 20 afirmativas que mensuram o grau de identificação do respondente com determinados comportamentos e sentimentos a partir de intervalo de cinco pontos, sendo: " 1 = Não é verdade"; " 2 = Raramente"; " 3 = Às vezes"; " $4=$ Frequentemente"; e " $5=$ Muito verdadeiro".

A CIPS é a escala de mensuração mais utilizada para a identificação do Fenômeno Impostor, sua pontuação pode variar de 20 a 100 pontos quando totalmente preenchida. Quanto mais alta a pontuação, mais intensas são as experiências e os sentimentos impostores experimentados pelo respondente (Clance, 1985). Indivíduos que obtêm 40 pontos ou menos, têm poucas características do FI. Entre 41 a 60 pontos há sentimentos impostores de forma moderada. De 61 a 80 pontos, os sentimentos impostores são experimentados frequentemente. Enquanto de 81 a 100 há intensas experiências impostoras. Além disso, foi disponibilizado um bloco com perguntas de caracterização dos respondentes.

Inicialmente, o projeto de pesquisa foi submetido e aprovado pelo Comitê de Ética em Pesquisa da instituição a qual os pesquisadores estão vinculados e foi registrado na Plataforma Brasil sob o número CCAE 95480818.9.0000.0102. No questionário foi disponibilizada uma pergunta filtro para identificar respondentes que não fossem pós-graduandos stricto sensu, além de uma questão para identificar a área do conhecimento a que pertencia o curso do respondente.

As análises quantitativas realizadas no decorrer dos pré-testes e da coleta final seguiram o Protocolo de Análise Estatística exposto na Figura 2. 


\begin{tabular}{|c|c|c|}
\hline Indicadores & Parâmetro & Suporte Teórico \\
\hline \multicolumn{3}{|c|}{ Análise da Confiabilidade da Escala } \\
\hline Alpha de Cronbach & $\begin{aligned} & \geq 0,70: \text { Ideal } \\
\geq 0,60 & <0,70: \text { Satisfatório }\end{aligned}$ & $\begin{array}{l}\text { Hair Jr, Black, Babin, Anderson, } \\
\text { e Tatham (2009) }\end{array}$ \\
\hline \multicolumn{3}{|c|}{ Análise Fatorial Confirmatória (AFC) } \\
\hline$\chi^{2}$ & Quanto menor, melhor & \multirow{8}{*}{ Marôco (2014) } \\
\hline$p$-value & $>0,050$ & \\
\hline $\mathrm{CFI}$ & $\begin{array}{c}<0,8: \text { Mau } \\
\geq 0,8<0,9: \text { Sofrível } \\
\geq 0,9<0,95: \text { Bom } \\
\geq 0,95: \text { Ótimo }\end{array}$ & \\
\hline PCFI & $\begin{array}{c}\text { <0,60: Mau } \\
\geq 0,60<0,8: \text { Bom } \\
\geq 0,8: \text { Ótimo }\end{array}$ & \\
\hline GFI & $\begin{array}{c}<0,8: \text { Mau } \\
\geq 0,8<0,9: \text { Sofrível } \\
\geq 0,9<0,95: \text { Bom } \\
\geq 0,95: \text { Ótimo }\end{array}$ & \\
\hline PGFI & $\begin{array}{c}\text { <0,60: Mau } \\
\geq 0,60<0,8: \text { Bom } \\
\geq 0,8: \text { Ótimo }\end{array}$ & \\
\hline RMSEA & $\begin{array}{l}>0,10: \text { Inaceitável } \\
>0,05 ; 0,10 \leq \text { : Bom } \\
\leq 0,05 \text { : Muito bom }\end{array}$ & \\
\hline MECVI & Quanto menor, melhor & \\
\hline Cargas fatoriais & $\begin{array}{l}\text { Desejável: acima de } 0,50 \\
\text { Admissível: acima de } 0,40\end{array}$ & \multirow{3}{*}{ Hair Jr et al. (2009) } \\
\hline Comunalidades & De preferência acima de 0,50 & \\
\hline Variância Extraída Média & Acima de 0,50 & \\
\hline Confiabilidade Composta & Acima de 0,70 & \multirow{2}{*}{$\begin{array}{l}\text { Hair Jr et al. (2009) e Marôco } \\
\text { (2014) }\end{array}$} \\
\hline Validade Discriminante & $\begin{array}{l}\text { A Variância Média Extraída deverá ser maior que a } \\
\text { estimativa quadrática da correlação. }\end{array}$ & \\
\hline \multicolumn{3}{|c|}{ Análise Fatorial Exploratória (AFE) } \\
\hline $\begin{array}{l}\text { Medida de Adequação Amostral } \\
\text { (MAS) }\end{array}$ & $\begin{array}{c}\quad<0,50: \text { Inaceitável } \\
\geq 0,50<0,60: \text { Ruim } \\
\geq 0,60<0,70: \text { Medíocre } \\
\geq 0,70<0,80: \text { Mediano } \\
\text { > 0,80: Admirável }\end{array}$ & Hair Jr et al. (2009) \\
\hline KMO & 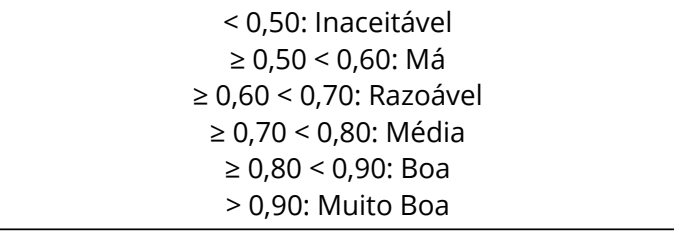 & Fávero e Belfiore (2017) \\
\hline Teste de Bartlett & $p$-value $<0,050$ & \multirow{3}{*}{ Field (2009) e Hair Jr et al. (2009) } \\
\hline Comunalidades & $\begin{array}{l}\text { De preferência acima de } 0,50 \text {. Aceitáveis acima de } \\
0,30 \text {. }\end{array}$ & \\
\hline Cargas fatoriais & $\begin{array}{l}\text { Desejável: acima de } 0,50 \\
\text { Admissível: acima de } 0,40\end{array}$ & \\
\hline Retenção dos fatores & Acima de $50 \%$ & Marôco (2007) \\
\hline
\end{tabular}

Figura 2. Protocolo de Análise Estatística

Nota. $\chi 2$ = Qui-quadrado; CFI = Comparative Fit Index; PCFI = Parcimony Comparative Fit Index; GFI = Goodness-of-Fit Index;

RMSEA = Root Mean Square Error of Approximation; MECVI = Modified Expected Cross-Validation Index; KMO = Kaiser-Meyer-Olkin.

Fonte: elaborado pelos autores. 
A CIPS foi desenvolvida originalmente em inglês, sendo que no Brasil há algumas traduções difusas dessa escala. Perante isso, foi solicitada, via e-mail, autorização para tradução e uso da escala à autora. Depois de obtida a autorização, a escala foi submetida à tradução juramentada para ajustes posteriores. No Brasil, a tradução juramentada tem fé pública, fato que garante, ou aproxima ao máximo, que o conteúdo escrito em português reflete o conteúdo do documento escrito em língua estrangeira (ATPP, 2018). Após a tradução, o instrumento de pesquisa foi analisado por dois profissionais que lecionam no nível stricto sensu, sendo um da área de métodos quantitativos e outro de metodologia da pesquisa científica e que são pesquisadores vinculados a programas de pós-graduação da área de negócios. O objetivo desta verificação centrou-se na estrutura metodológica da escala, não sendo indicado nenhum ajuste.

Na Fase 1 do pré-teste, o questionário foi enviado a 592 pós-graduandos stricto sensu e pesquisadores de diferentes áreas de conhecimento e que não compõe a amostra final. Foram obtidas 113 participações, sendo 100 respostas válidas para serem analisadas. Além disso, 37 pós-graduandos preencheram o campo que foi destinado para sugestões e comentários inerentes ao instrumento de coleta de dados. Cada participação recebeu um código de identificação.

Inicialmente, foi realizada a análise da confiabilidade da escala pelo Alpha de Cronbach, em que a análise preliminar indicou que a escala possuía consistência interna, à medida que o Alpha de Cronbach apresentou grau de confiabilidade de 0,919 (Fávero \& Belfiore, 2017). Entretanto, Field (2009) alerta que o Alpha de Cronbach varia a partir do número de itens da escala, quanto maior a quantidade de itens, mais elevado tende a ser o coeficiente obtido, pois o numerador da equação eleva o número de itens da escala ao quadrado. Nesse sentido, foram também analisadas as sugestões realizadas pelos pós-graduandos stricto sensu.

Os pós-graduandos 14 e 27 indicaram que os rótulos das assertivas poderiam induzir as respostas, por exemplo, a afirmativa 1 inicia com a expressão "Eu frequentemente", sendo que " 4 = Frequentemente" é um dos rótulos da escala, essa expressão pode ter induzido e criado viés para alguns respondentes da pesquisa, prejudicando a consistência interna e a unidimensionalidade dos constructos. A partir disso foi realizada a verificação de cada assertiva e destacadas as expressões que pudessem enviesar as respostas, bem como calculados a média e o desvio-padrão de cada item para análise. Na Figura 3, são apresentadas as assertivas com expressões que poderiam causar viés de resposta.

\begin{tabular}{|c|c|c|}
\hline Assertivas & M & DP \\
\hline $\begin{array}{l}\text { 1. Eu frequentemente tenho sucesso em um teste ou tarefa, mesmo com medo de não me sair bem } \\
\text { antes de assumir a tarefa. }\end{array}$ & 3,848 & 0,813 \\
\hline $\begin{array}{l}\text { 5. Às vezes, acho que obtive minha posição atual o } \\
\text { no lugar certo, na hora certa, ou conhecia as pesso }\end{array}$ & 2,616 & 1,330 \\
\hline te faço um projeto ou uma tarefa tão bem qua & 2,808 & 1,322 \\
\hline $\begin{array}{l}\text { 9. Às vezes, sinto ou acredito que o sucesso em minha vida ou em meu trabalho é resultado de algum } \\
\text { tipo de erro. }\end{array}$ & 1,990 & 1,241 \\
\hline to que meu sucesso foi devido a algum & 2,424 & 1,270 \\
\hline 12. Às vezes, fico decepcionado com minhas realizações atuais e acho que deve & 3,323 & 1,194 \\
\hline tenho medo de que os outros descubram o quanto me falta de conhecimento ou habilidade. & 2,939 & 1,406 \\
\hline $\begin{array}{l}\text { 14. Muitas vezes, tenho medo de que eu possa falhar em uma nova tarefa ou empreendimento, } \\
\text { embora eu geralmente faça bem o que tento. }\end{array}$ & 3,354 & 1,189 \\
\hline $\begin{array}{l}\text { 17. Eu frequentemente comparo minha habilidade com aqueles que estão ao meu redor e penso que } \\
\text { eles podem ser mais inteligentes do que eu. }\end{array}$ & 3,455 & 1,272 \\
\hline $\begin{array}{l}\text { 18. Muitas vezes me preocupo em não ter sucesso com um projeto ou exame, mesmo que outros à } \\
\text { minha volta tenham confiança considerável de que eu terei sucesso. }\end{array}$ & 3,303 & 1,173 \\
\hline
\end{tabular}

Figura 3. Clance Imposter Phenomenon Scale - CIPS

Nota. M = Média; DP = Desvio Padrão.

Fonte: Clance (1985). 
Por meio da média, do desvio-padrão e do boxplot de cada item foi possível verificar a dispersão das assertivas que compõem a CIPS, sendo identificadas afirmativas com respostas concentradas nas opções que condiziam o início da afirmativa com o rótulo da escala, a exemplo da assertiva "1. Eu frequentemente tenho sucesso em um teste ou tarefa, mesmo com medo de não me sair bem antes de assumir a tarefa". Dessa forma, foram modificadas ou excluídas as expressões das afirmativas 1, 8, 9, 11, 12,13 14, 17 e 18 que pudessem interferir e direcionar a interpretação dos participantes. A afirmativa 5 foi ajustada para facilitar a fluidez do texto, conforme sugestão do pós-graduando 100. Todas as modificações foram realizadas após discussões conjuntas com um professor e pesquisador da área de metodologia da pesquisa científica, com vistas à manutenção do conteúdo e sentido das assertivas.

$\mathrm{Na}$ Fase 2 do pré-teste, foram enviados convites para participação do pré-teste por e-mail a 453 coordenações de programas de pós-graduação de diferentes áreas do conhecimento solicitando a divulgação do pré-teste aos discentes, e, de modo adicional, foram convidados, via e-mail, 1.316 pósgraduandos stricto sensu para colaborar com esta fase da pesquisa. Totalizaram-se 558 participações, sendo 513 participações válidas para análise. Foram obtidas participações de discentes de 99 programas de diferentes subáreas do conhecimento. Após a tabulação dos dados, foi analisado o nível de confiabilidade da escala por meio do Alpha de Cronbach que indicou um valor de 0,921.

Em face às limitações do Alpha de Cronbach, seguiu-se a verificação da Análise Fatorial Confirmatória e Análise Fatorial Exploratória para identificar a unidimensionalidade dos constructos, operacionalizadas por meio dos softwares IBM SPSS AMOS v. 21.0.0 e IBM SPSS Statistics v. 19.1 ${ }^{\circledR}$. Inicialmente, foi utilizada a Análise Fatorial Confirmatória e foi verificada a existência de outliers a partir do quadrado da distância de Mahalanobis e do p1 e p2 menores que 0,001. Apesar de oito observações apresentarem p1 e p2 não satisfatórios, o quadrado da distância de Mahalanobis não apresentou distanciamento elevado que pudesse indicar a necessidade de exclusão das observações. 
Em relação às assertivas, nenhuma variável apresentou violação extrema da normalidade que pudesse indicar em um primeiro momento a necessidade de exclusão, sendo todos os valores obtidos para a assimetria ( $s k)$ abaixo de 2 e a curtose $(k u)$ abaixo de 7 , conforme critérios definidos por Marôco (2014). Na Tabela 1, são apresentados os valores de assimetria e curtose de cada variável do Fenômeno Impostor e os indicadores de ajustamento.

Tabela 1

Assimetria (sk) e curtose (ku) - AFC

\begin{tabular}{cccccc}
\hline Variável & Assimetria (sk) & Curtose (ku) & Variável & Assimetria (sk) & Curtose (ku) \\
\hline$F 1$ & $-0,695$ & 1,454 & $F 11$ & 0,730 & $-0,706$ \\
\hline$F 2$ & $-0,343$ & $-0,585$ & $F 12$ & $-0,152$ & $-1,112$ \\
\hline$F 3$ & $-0,018$ & $-0,983$ & $F 13$ & $-0,101$ & $-1,218$ \\
\hline$F 4$ & $-0,404$ & $-1,055$ & $F 14$ & $-0,479$ & $-0,554$ \\
\hline$F 5$ & 0,613 & $-1,031$ & $F 15$ & 0,008 & $-1,072$ \\
\hline$F 6$ & 0,066 & $-1,305$ & $F 16$ & 0,260 & $-1,229$ \\
\hline$F 7$ & $-0,288$ & $-0,996$ & $F 17$ & $-0,358$ & $-0,984$ \\
\hline$F 8$ & $-0,043$ & $-0,884$ & $F 18$ & $-0,464$ & $-0,612$ \\
\hline$F 9$ & 0,793 & $-0,475$ & $F 19$ & $-0,805$ & $-0,329$ \\
\hline$F 10$ & 0,005 & -1.146 & $F 20$ & $-0,198$ & $-0,953$ \\
\hline Indicador & \multicolumn{7}{c}{ Interpretação } & Indicador & & Interpretação \\
\hline$\chi 2$ & $1.138,544$ & Parâmetro não atendido & GFI & 0,799 & Mau \\
\hline$p$-value & 0,000 & Parâmetro não atendido & PGFI & 0,647 & Bom \\
\hline CFI & 0,816 & Sofrível & RMSEA & 0,105 & Inaceitável \\
\hline PCFI & 0,730 & Bom & MECVI & 2,387 & Ajustamento ruim \\
\hline
\end{tabular}

Fonte: elaborado pelos autores.

A partir da Tabela 1, percebeu-se que o ajustamento de alguns indicadores não estava adequado, pois não apresentavam parâmetros satisfatórios, conforme exposto no protocolo de análise. Ao verificar as cargas fatoriais padronizadas, foram identificadas assertivas com valores inferiores a 0,50 , sugerindo que mais da metade da explicação do item se refere aos erros e não à variação do item em si (Hair Jr et al., 2009). As assertivas com valores abaixo do parâmetro de Hair Jr et al. (2009) são destacadas na Tabela 2.

Tabela 2

Cargas Fatoriais Padronizadas - AFC

\begin{tabular}{|c|c|c|c|c|c|c|c|}
\hline ID & $\begin{array}{l}\text { Cargas Fatoriais } \\
\text { Padronizadas }\end{array}$ & ID & $\begin{array}{c}\text { Cargas Fatoriais } \\
\text { Padronizadas }\end{array}$ & ID & $\begin{array}{c}\text { Cargas Fatoriais } \\
\text { Padronizadas }\end{array}$ & ID & $\begin{array}{c}\text { Cargas Fatoriais } \\
\text { Padronizadas }\end{array}$ \\
\hline $\mathrm{F} 1$ & $-0,098$ & F6 & 0,764 & F11 & 0,700 & F16 & 0,669 \\
\hline F2 & 0,221 & F7 & 0,682 & F12 & 0,686 & F17 & 0,706 \\
\hline F3 & 0,601 & F8 & 0,596 & F13 & 0,792 & F18 & 0,697 \\
\hline F4 & 0,723 & F9 & 0,715 & F14 & 0,730 & F19 & 0,215 \\
\hline F5 & 0,624 & F10 & 0,644 & F15 & 0,826 & $\mathrm{~F} 20$ & 0,384 \\
\hline \multicolumn{4}{|c|}{ Confiabilidade Composta $(C C)=0,9221$} & \multicolumn{4}{|c|}{ Variância Média Extraída $(\mathrm{VME})=0,519$} \\
\hline
\end{tabular}

Fonte: elaborado pelo autor. 
Apesar da Variância Média Extraída ter apresentado valor ligeiramente superior ao adequado e da Confiabilidade Composta ter sido satisfatória, optou-se por analisar a origem das baixas cargas fatoriais padronizadas das assertivas que apresentaram piores resultados, nesse caso a F1, F2, F19 e F20, a fim de melhorar os indicadores de ajustamento do modelo. A partir de uma busca na literatura, foram identificados estudos que aplicaram a AFC e AFE na CIPS (Chrisman, Pieper, Clance, Holland, \& GlickaufHughes, 1995; French et al., 2008) e que encontraram problemas semelhantes com os itens 1, 2, 19 e 20, sugerindo a exclusão deles e a verificação da existência de dois (French et al., 2008) ou três (Chrisman et al., 1995) fatores oriundos das assertivas restantes.

Logo, após a exclusão dos quatro itens foi aplicada a AFE na CIPS, a fim de analisar a estrutura fatorial retornada. Com a utilização do método de extração Componentes Principais e método de rotação Varimax, foram retornados dois fatores com $60,52 \%$ de explicação da variância total. Optou-se pelo método Varimax, com o intuito de maximizar a soma das variâncias das cargas exigidas da matriz fatorial (Hair Jr et al., 2009). As correlações anti-imagem foram maiores que 0,70 na diagonal, o KMO foi de 0,946, o teste de Bartlett apresentou significância e as comunalidades apresentaram indicadores superiores ou próximos a 0,50. Conforme sugerido por Hair Jr et al. (2009), foram ocultadas as cargas fatoriais menores que 0,40 , por não serem significantes. Na Tabela 3 , é apresentada a adequação das variáveis em seus fatores.

Tabela 3

Adequação das variáveis aos fatores - AFE - CIPS

\begin{tabular}{|c|c|c|c|c|c|c|c|}
\hline \multicolumn{6}{|c|}{ Fator 1} & \multicolumn{2}{|r|}{ Fator 2} \\
\hline ID & Cargas Fatoriais & ID & Cargas Fatoriais & ID & Cargas Fatoriais & ID & Cargas Fatoriais \\
\hline F14 & 0,778 & $\mathrm{~F} 4$ & 0,718 & F10 & 0,627 & FI11 & 0,875 \\
\hline F13 & 0,770 & F7 & 0,657 & F16 & 0,61 & $\mathrm{FI9}$ & 0,856 \\
\hline F15 & 0,767 & $\mathrm{~F} 12$ & 0,637 & F3 & 0,563 & $\mathrm{Fl} 5$ & 0,831 \\
\hline F18 & 0,767 & F6 & 0,631 & F8 & 0,492 & & \\
\hline F17 & 0,724 & & & & & & \\
\hline
\end{tabular}

Nota. ID = Identificação.

Fonte: elaborado pelo autor. 
Assim como no trabalho de French et al. (2008), foram identificados dois fatores, sendo o "Fator 1 - Falsidade e Subestimação", alinhado às assertivas de falsidade e subestimação das próprias habilidades, e o "Fator 2 - Sorte ou Acaso", inerente às crenças de que o sucesso obtido foi devido ao acaso e a sorte. Além disso, as assertivas F6 e F8 compartilham cargas com mais de um fator, sendo optado por manter tais assertivas, a fim de não prejudicar a validade do modelo. Uma nova AFC foi realizada em conjunto com os dois fatores encontrados, sendo obtidos resultados satisfatórios nos indicadores de ajustamento, Confiabilidade Composta, Variância Média Extraída e correlação entre os fatores, conforme Tabela 4.

Tabela 4

Indicadores de ajustamento - AFC

\begin{tabular}{|c|c|c|c|c|c|c|}
\hline \multicolumn{2}{|c|}{ Indicador } & \multirow{2}{*}{$\begin{array}{c}\text { Interpretação } \\
\text { Parâmetro atendido }\end{array}$} & \multicolumn{2}{|c|}{ Indicador } & \multirow{2}{*}{\multicolumn{2}{|c|}{$\begin{array}{c}\text { Interpretação } \\
\text { Bom }\end{array}$}} \\
\hline$\chi^{2}$ & 390,089 & & GFI & 0,911 & & \\
\hline$p$-value & 0,000 & Parâmetro não atendido & PGFI & 0,690 & & \\
\hline CFI & 0,943 & Bom & RMSEA & 0,074 & & \\
\hline PCFI & 0,810 & Bom & MECVI & 0,895 & Ajusta & o bom \\
\hline \multicolumn{5}{|c|}{ Correlação entre Fator 1 com Fator 2} & \multicolumn{2}{|c|}{0,696} \\
\hline \multicolumn{5}{|c|}{ Validade Discriminante (VD) } & \multicolumn{2}{|c|}{0,484} \\
\hline & & & & & Fator 1 & Fator 2 \\
\hline \multicolumn{5}{|c|}{ Confiabilidade Composta (CC) } & 0,9292 & 0,9149 \\
\hline \multicolumn{5}{|c|}{ Variância Média Extraída (VME) } & 0,5046 & 0,7825 \\
\hline
\end{tabular}

Fonte: elaborado pelo autor.

Compararam-se os indicadores de ajustamento desta pesquisa aos encontrados por French et al. (2008) no modelo de dois fatores. Na pesquisa de French et al. (2008), obtiveram-se: $\chi^{2}=1472,85, p$-value $<$ $0,050, \mathrm{CFI}=0,796$. Desse modo, o único parâmetro não atendido foi o $p$-value do $\chi^{2}$, assim como French et al. (2008), sendo que os demais indicadores obtiveram bons resultados e ajustamento melhor que o de French et al. (2008). As assertivas F5, F8 e F11 conseguem explicar 78,25\% da variância do "Fator 2 - Sorte", enquanto as demais assertivas explicam $50,46 \%$ do "Fator 1 - Falsidade e Subestimação". Os fatores não estão altamente correlacionados, bem como a Validade Discriminante é confirmada. A capacidade explicativa de tais fatores pode ser alterada na amostra final, em face das diferentes características da amostra, e fornecem evidências da estrutura e dos direcionamentos para utilização da CIPS na próxima fase da pesquisa. Perante a viabilidade do modelo, foi utilizada a CIPS com 16 assertivas na coleta final. A versão após os ajustes foi encaminhada para a autora da escala original e é apresentada na análise dos resultados. Após serem finalizadas as fases do pré-teste, foi realizada a coleta de dados final com pós-graduandos da área de negócios. 


\section{Resultados}

Foram obtidas 2.259 participações, sendo que 1.816 foram consideradas válidas para serem analisadas. Destes respondentes, $51,21 \%$ se identificaram como sendo do gênero feminino; os respondentes estão na faixa etária de 21 a 66 anos; $2,42 \%$ estão vinculados a instituições de ensino da região Norte, 7,71\% Centro-Oeste, $14,98 \%$ do Nordeste, $28,64 \%$ do Sul e $46,25 \%$ do Sudeste.

Na Tabela 5, são expostas as 16 assertivas do Fenômeno Impostor, restantes após a exclusão da F1, F2, F19 e F20 realizada na fase do pré-teste, bem como a estatística descritiva.

Tabela 5

\section{Clance Impostor Phenomenon Scale - Estatística descritiva}

\begin{tabular}{|c|c|c|c|c|}
\hline Assertiva & M & Mo & Md & Dp \\
\hline $\begin{array}{l}\text { F18 - Me preocupo em não ter sucesso com um projeto ou avaliação, mesmo que outros } \\
\text { à minha volta tenham confiança considerável de que eu terei sucesso. }\end{array}$ & 2,992 & 3 & 3 & 1,249 \\
\hline $\begin{array}{l}\text { F7 - Tenho a tendência de lembrar mais os incidentes em que não fiz o melhor que pude } \\
\text { do que os momentos em que fiz o melhor que pude. }\end{array}$ & 2,843 & 3 & 3 & 1,252 \\
\hline $\begin{array}{l}\text { F14 - Tenho medo de que eu possa falhar em uma nova tarefa ou empreendimento, } \\
\text { embora eu geralmente faça bem o que tento. }\end{array}$ & 2,831 & 3 & 3 & 1,194 \\
\hline $\begin{array}{l}\text { F17 - Eu comparo minhas habilidades com aqueles que estão ao meu redor e penso que } \\
\text { eles podem ser mais inteligentes do que eu. }\end{array}$ & 2,831 & 3 & 3 & 1,275 \\
\hline F8 - Dificilmente eu faço um projeto ou uma tarefa tão bem quanto eu gostaria. & 2,733 & 3 & 3 & 1,187 \\
\hline $\begin{array}{l}\text { F12 - Fico decepcionado com minhas realizações atuais e acho que deveria ter feito } \\
\text { muito mais. }\end{array}$ & 2,681 & 3 & 3 & 1,318 \\
\hline $\begin{array}{l}\text { F4 - Quando as pessoas me elogiam por algo que eu realizei, tenho medo de não } \\
\text { corresponder às suas expectativas em relação à mim no futuro. }\end{array}$ & 2,593 & 2 & 3 & 1,220 \\
\hline $\begin{array}{l}\text { F6 - Tenho medo que as pessoas importantes para mim possam descobrir que eu não } \\
\text { sou tão capaz quanto elas pensam que sou. }\end{array}$ & 2,386 & 1 & 2 & 1,308 \\
\hline $\begin{array}{l}\text { F13 - Tenho medo de que os outros descubram o quanto me falta de conhecimento ou } \\
\text { habilidade. }\end{array}$ & 2,366 & 1 & 2 & 1,256 \\
\hline F10 - É difícil aceitar elogios ou cumprimentos sobre minha inteligência ou realizações. & 2,358 & 1 & 2 & 1,216 \\
\hline F3 - Se possível, eu evito avaliações e tenho medo de que outras pessoas me avaliem. & 2,299 & 3 & 2 & 1,065 \\
\hline $\begin{array}{l}\text { F15 - Quando consigo algo e recebo reconhecimento por minhas realizações, tenho } \\
\text { dúvidas de que posso continuar repetindo esse sucesso. }\end{array}$ & 2,275 & 1 & 2 & 1,158 \\
\hline $\begin{array}{l}\text { F16 - Se eu receber muitos elogios e reconhecimento por algo que realizei, tenho a } \\
\text { tendência de desconsiderar a importância do que fiz. }\end{array}$ & 2,216 & 1 & 2 & 1,229 \\
\hline $\begin{array}{l}\text { F5 - Acho que obtive minha posição atual ou obtive meu sucesso atual por acaso, porque } \\
\text { estava no lugar certo, na hora certa, ou conhecia as pessoas certas. }\end{array}$ & 1,818 & 1 & 1 & 1,129 \\
\hline F11 - Sinto que meu sucesso foi devido a algum tipo de sorte. & 1,718 & 1 & 1 & 0,984 \\
\hline $\begin{array}{l}\text { F9 - Sinto ou acredito que o sucesso em minha vida ou em meu trabalho é res } \\
\text { algum tipo de acaso. }\end{array}$ & 1,687 & 1 & 1 & 0,973 \\
\hline
\end{tabular}

Nota. $\mathrm{M}=$ Média; $\mathrm{Mo}=$ Moda; $\mathrm{Md}=$ Mediana; $\mathrm{Dp}=$ Desvio Padrão.

Fonte: Elaborado pelos autores. 
O intervalo da escala CIPS oscilou de 1 a 5 , no qual nenhum dos itens alcançou média igual ou superior a 3, as três maiores e menores médias são comentadas adiante. O item com maior média foi "F18 - Me preocupo em não ter sucesso com um projeto ou avaliação, mesmo que outros à minha volta tenham confiança considerável de que eu terei sucesso" $(\mathrm{M}=2,992 ; \mathrm{DP}=1,249)$, tendo como opção mais assinalada a "3 = Às vezes" ( $\mathrm{Mo}=3 ; \mathrm{Md}=3)$. O F18 versa sobre a insegurança do indivíduo frente à sua capacidade de alcançar sucesso em determinadas tarefas, este aspecto figura entre as principais características dos impostores, já retratadas no trabalho seminal de Clance e Imes (1978) no qual mulheres de prestígio eram inseguras diante das suas competências. Nesse sentido, mesmo a amostra sendo composta de pessoas com elevado grau de instrução, mestrandos ou doutorandos, nota-se que há um nível elevado de medo inerente à obtenção de sucesso no alcance dos objetivos. Esses sentimentos podem ser maximizados ao observar o ambiente da pós-graduação que tem sido caracterizado como complexo, gerador de inseguranças e pressões sobre os discentes (Levecque, Anseel, De Beuckelaer, Van der Heyden, \& Gisle, 2017).

A "F7 - Tenho a tendência de lembrar mais os incidentes em que não fiz o melhor que pude do que os momentos em que fiz o melhor que pude" $(\mathrm{M}=2,843 ; \mathrm{DP}=1,252)$ figurou como item de segunda maior média, com o rótulo " 3 = Às vezes" ( $\mathrm{MO}=3 ; \mathrm{MD}=3$ ), sendo o mais apontado pelos discentes. Dudău (2014) já havia identificado esta característica em estudantes romenos do curso de graduação em Psicologia, no qual indivíduos com sentimentos impostores tendem a dedicar maior atenção aos erros cometidos do que ao sucesso alcançado. Thompson et al. (1998) citam que a assimilação da capacidade intelectual tende a ser maximizada com o tempo e o acúmulo de objetivos alcançados, entretanto na maioria das vezes este processo é lento (Clance \& Imes, 1978), colaborando para que discentes, mesmo com níveis elevados de formação, se sintam impostores. No âmbito do stricto sensu, incentivar conversas em grupos, por meio do compartilhamento de relatos e vivências pode auxiliar discentes com sentimentos impostores a assimilar seus sucessos e capacidades intelectuais.

As assertivas "F14 - Tenho medo de que eu possa falhar em uma nova tarefa ou empreendimento, embora eu geralmente faça bem o que tento" $(\mathrm{M}=2,831$; $\mathrm{DP}=1,194)$ e "F17 - Eu comparo minhas habilidades com aqueles que estão ao meu redor e penso que eles podem ser mais inteligentes do que eu" $(\mathrm{M}=2,831 ; \mathrm{DP}=1,275)$ foram as assertivas com a terceira maior média e com a opção " 3 = Às vezes" $(\mathrm{MO}=3 ; \mathrm{MD}=3)$ sendo a mais assinalada. Em relação à F14, Holmes et al. (1993) alertam que indivíduos com esse comportamento podem recusar oportunidades de crescimento profissional e priorizar posições de baixo nível, aquém de suas habilidades, para evitar julgamentos alheios. Sobre a F17, no estudo de Craddock et al. (2011), os autores retrataram esta característica como preponderante em doutorandos com sentimentos impostores, visto que a sensação de não possuir o mesmo nível de capacidade intelectual que os colegas implica em sentimentos de não pertencimento ao ambiente da pós-graduação. Além disso, a competitividade acadêmica existente na pós-graduação (Levecque et al., 2017) pode favorecer o afloramento destes sentimentos.

Referente às assertivas com menores médias tem-se a "F9 - Sinto ou acredito que o sucesso em minha vida ou em meu trabalho é resultado de algum tipo de acaso" $(\mathrm{M}=1,687 ; \mathrm{DP}=0,973 ; \mathrm{MO}=1$; $\mathrm{MD}=1)$, seguida da "F11 - Sinto que meu sucesso foi devido a algum tipo de sorte" $(\mathrm{M}=1,718 ; \mathrm{DP}=$ 0,$984 ; \mathrm{MO}=1 ; \mathrm{MD}=1$ ), e, por fim, a "F5 - Acho que obtive minha posição atual ou obtive meu sucesso atual por acaso, porque estava no lugar certo, na hora certa, ou conhecia as pessoas certas" $(\mathrm{M}=1,818$; $\mathrm{DP}=1,129 ; \mathrm{MO}=1 ; \mathrm{MD}=1)$. Estas assertivas caracterizam a percepção de que o sucesso alcançado está relacionado a fatores supersticiosos. Nesse contexto, apesar de uma das características dos impostores seja relacionar suas conquistas à sorte (Clance \& Imes, 1978; Holmes et al., 1993), este aspecto não se mostrou preponderante na amostra analisada. 
A partir da soma das pontuações obtidas por cada discente no preenchimento da CIPS, foi elaborada a Tabela 6, que classifica os respondentes de acordo com o nível de sentimentos impostores. No estudo de Clance (1985) é explanada a versão original da CIPS com 20 assertivas, o intervalo original de pontuação desta versão varia de 20 a 100 pontos, pelo fato de a escala ter sido reduzida para 16 assertivas neste estudo, o intervalo varia de 16 a 80 pontos.

Tabela 6

Faixa de pontuação de sentimentos impostores

\begin{tabular}{ccccccc}
\hline Faixa & Descrição & F & \% & Faixa & Descrição & F \\
\hline $\begin{array}{l}16 \text { a } 32 \\
\text { pontos }\end{array}$ & $\begin{array}{c}\text { Poucas características do } \\
\text { Fenômeno Impostor. }\end{array}$ & 676 & 37,22 & $\begin{array}{l}49 \text { a } 64 \\
\text { pontos }\end{array}$ & Fl é frequente. \\
\hline $\begin{array}{l}\text { 33 a } 48 \\
\text { pontos }\end{array}$ & Fl é moderado. & 721 & 39,70 & $\begin{array}{l}65 \text { a } 80 \\
\text { pontos }\end{array}$ & $\begin{array}{c}\text { Fl é intenso e afeta a } \\
\text { assimilação de sucesso e }\end{array}$ \\
\hline
\end{tabular}

Nota. F = frequência; $\%$ = percentual.

Fonte: Elaborado pelos autores.

A maioria dos pós-graduandos da área de negócios participantes da pesquisa experimentam sentimentos impostores de forma moderada, neste grupo encontra-se 721 respondentes que representam $39,70 \%$ da amostra. Em seguida, tem-se o grupo dos discentes com poucas características do Fenômeno Impostor, com 676 pós-graduandos que representam $37,22 \%$ da amostra. O terceiro grupo é composto por 352 participantes, que representam 19,38\% dos respondentes da pesquisa e vivenciam frequentemente sentimentos impostores. Por fim, no grupo de pessoas que vivenciam intensas experiências impostoras tem-se 67 respondentes que consubstanciam em 3,69\% da amostra da pesquisa.

Após a análise descritiva, foi verificada a formação dos fatores da CIPS por meio da AFE, sendo que na Tabela 7 são apresentadas as comunalidades e os indicadores de adequação obtidos nesta etapa.

Tabela 7

AFE - Comunalidades e Indicadores de Adequação

\begin{tabular}{|c|c|c|c|c|c|c|c|}
\hline ID & Extração & ID & Extração & ID & Extração & ID & Extração \\
\hline F3 & 0,296 & F7 & 0,496 & F11 & 0,832 & F15 & 0,686 \\
\hline $\mathrm{F} 4$ & 0,523 & F8 & 0,449 & F12 & 0,483 & F16 & 0,467 \\
\hline F5 & 0,712 & F9 & 0,813 & F13 & 0,670 & $\mathrm{~F} 17$ & 0,525 \\
\hline F6 & 0,633 & F10 & 0,455 & F14 & 0,647 & F18 & 0,588 \\
\hline \multicolumn{2}{|c|}{ Indicador } & \multicolumn{2}{|c|}{ Interpretação } & \multicolumn{3}{|c|}{ Indicador } & Interpretação \\
\hline MAS mínimo & 0,863 (FI9) & \multirow{2}{*}{\multicolumn{2}{|c|}{ Admirável }} & \multirow{2}{*}{\multicolumn{2}{|c|}{ Bartlett Sig. }} & $16.197,32$ & \multirow{2}{*}{ Muito bom } \\
\hline MAS máximo & $0,976(\mathrm{FI} 5)$ & & & & & 0,000 & \\
\hline $\mathrm{KMO}$ & 0,944 & \multicolumn{2}{|c|}{ Muito boa } & & & & \\
\hline
\end{tabular}

Nota. ID = Identificação.

Fonte: elaborado pelos autores. 
Somente a assertiva "F3 - Se possível eu evito avaliações e tenho medo de que outras pessoas me avaliem" possui valor de comunalidade inferior a 0,30, a qual se abarca em 0,296. Entretanto, optou-se por não eliminar esta assertiva pelo valor ser muito próximo ao critério estabelecido, pelos fatores seguirem a mesma estrutura de formação do pré-teste e obtido no estudo de French et al. (2008); e pelos demais indicadores apresentarem-se apropriados.

Nota-se que os indicadores da MAS, obtidos por meio das correlações diagonais da matriz antiimagem, são adequados, sendo maiores que 0,70 e superiores às demais correlações da variável. Além disso, o KMO apresentou valor satisfatório $(0,944)$, bem acima do critério de 0,70 , assim como o teste de Bartlett que foi significante, indicando haver presença de correlações significantes entre as variáveis. Desse modo, a formação dos fatores é visualizada na Tabela 8, bem como a variância explicada por essa estrutura fatorial.

Tabela 8

AFE - Cargas fatoriais e variância explicada

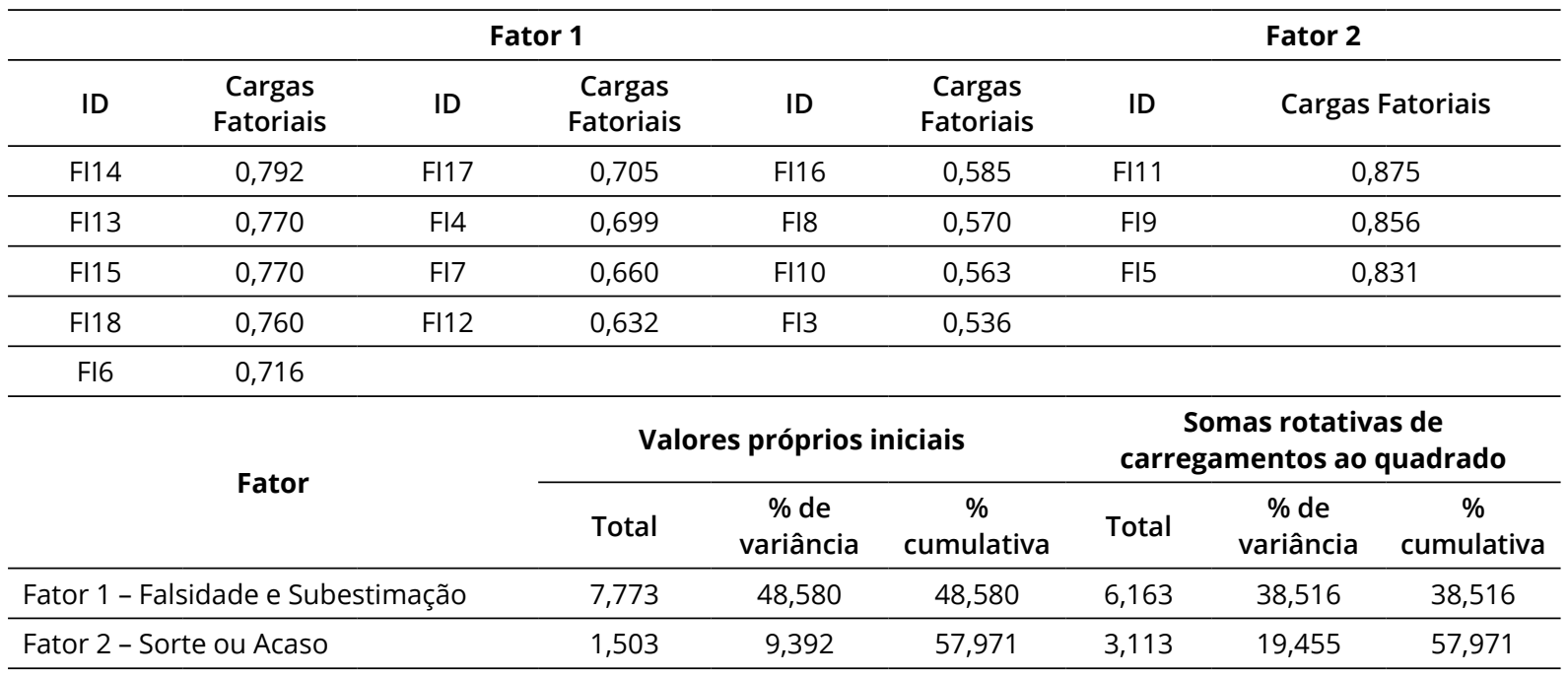

Nota. ID = Identificação

Fonte: elaborado pelos autores.

Percebe-se que assim como no estudo de French et al. (2008), foram formados dois fatores, sendo que as assertivas F3, F4, F6, F7, F8, F10, F12, F13, F14, F15, F16, F17 e F18 compõem "Fator 1 - Falsidade e Subestimação" com Alfa de Cronbach de 0,922, enquanto as assertivas F5, F9 e F11 são responsáveis por representar o "Fator 2 - Sorte ou Acaso" com Alfa de Cronbach de 0,869. Conforme defendido por Diamantopoulos e Siguaw (2000), o número mínimo de três variáveis por fator foi alcançado.

O Fator 1 foi denominado de "Falsidade e Subestimação", pois representa sentimentos de farsa e dúvidas dos indivíduos frente às suas capacidades de alcançar o sucesso. Enquanto ao Fator 2 foi atribuída a nomenclatura "Sorte ou Acaso", visto que é constituído pelas assertivas que indicam as atribuições de sucesso a situações aleatórias, relacionadas ao destino ou às coincidências. A estrutura fatorial obtida consegue explicar 57,971\% da variância dos dados originais. Nesse sentido, o "Fator 1 - Falsidade e Subestimação" é responsável por explicar $48,580 \%$ da variação dos sentimentos impostores, enquanto o "Fator 2 - Sorte ou Acaso" explica 9,392\% da variação. 
Com isso, o objetivo da pesquisa pode ser respondido da seguinte forma: a CIPS, quando analisada de forma bidimensional, apresenta indicadores satisfatórios. Ao analisar a amostra de interesse, nota-se que esta é caracterizada em sua maioria por discentes que vivenciam sentimentos impostores de forma moderada. Todas as características do Fenômeno Impostor são manifestadas por alguma parcela dos respondentes, sendo que estas características estão mais atreladas a sentimentos de dúvidas em relação à capacidade de obter sucesso, medo de falhar, subestimação das capacidades próprias e superestimação das capacidades dos colegas, visto que características relacionadas à sorte ou ao acaso não obtiveram, em geral, altas pontuações. A análise dos resultados ainda indicou que o Fenômeno Impostor presente na vida dos pós-graduandos stricto sensu da área de negócios pode ser dividido em dois grandes grupos, um que representa sentimentos de falsidade e subestimação e outro de sentimentos de sorte ou acaso.

\section{Conclusão}

Os achados indicam que sentimentos de dúvidas em relação à capacidade de obter sucesso, medo de falhar, subestimação das capacidades próprias e superestimação das capacidades dos colegas estão entre as principais características dos pós-graduandos stricto sensu da área de negócios que apresentam sentimentos impostores. Conforme descrito na literatura, esses sentimentos podem ser prejudiciais ao desenvolvimento dos discentes no ambiente acadêmico e no desenvolvimento de sua carreira profissional.

Para minimizar o Fenômeno Impostor, conversas em grupos podem auxiliar a pessoa a ver-se de forma mais realista, por meio do compartilhamento de relatos e vivências, e até mesmo a partir do registro de suas conquistas. Assim, controlar o Fenômeno Impostor torna-se fundamental para que o indivíduo possa consolidar sua autoestima e enfrentar os desafios de que permeiam o campo acadêmico e profissional de maneira mais segura, confiante e proativa.

A pesquisa ainda apresenta contribuições nos campos metodológico e teórico ao realizar a validação da Clance Impostor Phenomenon Score e identificar a sua bidimensionalidade, possibilitando que novas pesquisas sejam desenvolvidas. O estudo contribui em termos sociais ao discutir o FI, suas consequências sobre os pós-graduandos e indicar formas de minimizar tais sentimentos.

As limitações da pesquisa pairam na escassez de estudos desenvolvidos na área de negócios e no stricto sensu que possibilitem a comparação dos resultados, bem como na não aleatoriedade da amostra que impossibilita a generalização dos resultados para a população. Sugere-se que estudos futuros investiguem outras áreas do conhecimento para que o Fenômeno Impostor tenha suas características delineadas no stricto sensu.

\section{Referências}

Andrade, R. de O. (2018). À Procura Da Versatilidade. Retrieved July 16, 2018, from http://revistapesquisa. fapesp.br/wp-content/uploads/2018/02/094-097_carreiras_264.pdf

ATPP. (2018). O que é uma tradução juramentada? Retrieved July 24, 2018, from http://atpp.org.br/oque-e-traducao-juramentada/

Celebrity Insider (2017). Imposter Syndrome Prevented Renee Zellweger From Acting For 6 Years. Retrieved July 12, 2018, from https://celebrityinsider.org/imposter-syndrome-prevented-reneezellweger-from-acting-for-6-years-77337/

Cisco, J. (2020). Exploring the connection between impostor phenomenon and postgraduate students feeling academically-unprepared. Higher Education Research \& Development, 1-15. 
Chassangre, K., \& Callahan, S. (2017). «J'ai réussi, j’ai de la chance... je serai démasqué » : revue de littérature du syndrome de l'imposteur. Pratiques Psychologiques, 23(2), 97-110. Doi: https://doi. org/10.1016/j.prps.2017.01.00

Chassangre, K. (2014). Le phénomène de l'imposteur, la peur qui mine la réussite. Journal de Therapie Comportementale et Cognitive, 24(1), 32-38. Doi: https://doi.org/10.1016/j.jtcc.2013.12.001

Chrisman, S. M., Pieper, W. A., Clance, P. R., Holland, C. L., \& Glickauf-Hughes, C. (1995). Validation of the Clance Impostor Phenomenon Scale. Journal of Personality Assessment, 65(3), 456-467. Doi: https://doi.org/10.1207/s15327752jpa6503

Clance, P. R. (1985). The impostor phenomenon: Overcoming the fear that haunts your success. Atlanta, GA: Peachtree.

Clance, P. R., Dingman, D., Reviere, S., \& Stober, D. (1995). Impostor Phenomenon in an Interpersonal/ Social Context. Women \& Therapy, 16(4), 79-96. Doi: https://doi.org/10.1300/J015v16n04_07

Clance, P. R., \& Imes, S. A. (1978). The imposter phenomenon in high achieving women: Dynamics and therapeutic intervention. Psychotherapy: Theory, Research \& Practice, 15(3), 241-247. Doi: https:// doi.org/10.1037/h0086006

Clance, P. R., \& O’Toole, M. A. (1987). The Imposter Phenomenon. Women \& Therapy, 6(3), 51-64. Doi: https://doi.org/10.1300/J015V06N03_0

Craddock, S., Birnbaum, M., Rodriguez, K., Cobb, C., \& Zeeh, S. (2011). Doctoral Students and the Impostor Phenomenon: Am I Smart Enough to Be Here? Journal of Student Affairs Research and Practice, 48(4), 429-442. Doi: https://doi.org/10.2202/1949-6605.6321

Deffendall, M., Knutson, N., \& Sacks, D. (2011). A Profile on First- Generation College Students at the University of Kentucky: A Focus on Retention Efforts, 1-22. Retrieved from http://uknowledge. uky.edu/uge_reports\%5Cnhttp://uknowledge.uky.edu/uge_reports/1

Diamantopoulos, A., \& Siguaw, J. A. (2000). Introducing lisrel. London: Sage.

Entrepreneur. (2017). 12 Leaders, Entrepreneurs and Celebrities Who Have Struggled With Imposter Syndrome. Retrieved December 18, 2019, https://www.entrepreneur.com/slideshow/304273

Fávero, L. P., \& Belfiore, P. (2017). Manual de Análise de Dados - Estatística e Modelagem Multivariada com Excel $^{\oplus}$, SPSS $^{\circledast}$ e Stata ${ }^{\oplus}$ (1. ed.). Rio de Janeiro: Elsevier.

Ferrari, J. R., \& Thompson, T. (2006). Impostor fears: Links with self-presentational concerns and selfhandicapping behaviours. Personality and Individual Differences, 40(2), 341-352.

Field, A. (2009). Descobrindo a Estatística usando o SPSS (2. Ed.). Porto Alegre: Artmed.

Freire, C. (2014). Academic Misconduct Among Portuguese Economics and Business Undergraduate Students- A Comparative Analysis with Other Major Students. Journal of Academic Ethics, 12(1), 43-63. Doi: https://doi.org/10.1007/s10805-013-9199-2

French, B. F., Ullrich-French, S. C., \& Follman, D. (2008). The psychometric properties of the Clance Impostor Scale. Personality and Individual Differences, 44(5), 1270-1278.

Hair Jr, J. F., Black, W. C., Babin, B. J., Anderson, R. E., \& Tatham, R. L. (2009). Análise multivariada de dados. Porto Alegre: Bookman.

Holmes, S. W., Kertay, L., Adamson, L. B., Holland, C. L., \& Clance, P. R. (1993). Measuring the Impostor Phenomenon: A Comparison of Clance's IP Scale and Harvey's I P Scale. Journal of Personality Assessment, 60(1), 48-59. https://doi.org/10.1207/s15327752jpa6001 
Idiva (2019). Even Emma Watson Suffers From Imposter Syndrome, How Many Of You Can Relate? Retrieved December 18, 2019, from https://www.idiva.com/news-opinion/womens-issues/emmawatson-says-she-has-imposter-syndrome-and-so-many-of-us-can-relate/18004312

Kets de Vries, M. F. R. (1989). the Impostor Syndrome : a Disquieting Phenomenon in Organizational Life, 47.

Langford, J., \& Clance, P. R. (1993). The impostor phenomenon: Recent research findings regarding dynamics, personality and family patterns and their implications for treatment. Psychotherapy, 30(3), 495-501. Doi: https://doi.org/10.1037/0033-3204.30.3.495

Levecque, K., Anseel, F., De Beuckelaer, A., Van der Heyden, J., \& Gisle, L. (2017). Work organization and mental health problems in PhD students. Research Policy, 46(4), 868-879. Doi: https://doi. org/10.1016/j.respol.2017.02.008

Marie Claire. (2019). What Is Imposter Syndrome, And Why Do So Many Successful Women Suffer From It. Retrieved December 21, 2019, from https://www.marieclaire.com.au/imposter-sydnrome

Marôco, J. (2007). Análise Estatística com Utilização do SPSS. Lisboa: Edições Sílabo.

Marôco, J. (2014). Análise de Equações Estruturais - Fundamentos Teóricos, Software e Aplicações (2ª̂). Pêro Pinheiro: ReportNumber.

McCabe, D. L., Butterfield, K. D., \& Treviño, L. K. (2006). Academic dishonesty in graduate business programs: Prevalence, causes, and proposed action. Academy of Management Learning and Education, 5(3), 294-305. Doi: https://doi.org/10.5465/AMLE.2006.22697018

Sonnak, C., \& Towell, T. (2001). The impostor phenomenon in British university students: Relationships between self-esteem, mental health, parental rearing style and socioeconomic status. Personality and Individual Differences, 31(6), 863-874. Doi: https://doi.org/10.1016/S0191-8869(00)00184-7

Stone, S., Saucer, C., Bailey, M., Garba, R., Hurst, A., Jackson, S. M., Cokley, K. (2018). Learning While Black: A Culturally Informed Model of the Impostor Phenomenon for Black Graduate Students. Journal of Black Psychology. https://doi.org/10.1177/0095798418786648

Survey Monkey. (2021). Calculadora de tamanho de amostra. Retrieved September 10, 2021, from https:// pt.surveymonkey.com/mp/sample-size-calculator/

Taylor, A. (2009). The Impostor Phenomenon: A Look at the Outside, the Inside, and the Other Side Through Scholarly Personal Narrative. School of Education Doctor of Philosophy Colorado State University. Doctor of Philosophy - Colorado State University.

The Enterprisers Project. (2019). 5 ways to beat imposter syndrome. Retrieved December 18, 2019, from https://enterprisersproject.com/article/2019/8/imposter-syndrome-5-ways-beat

Thompson, T., Davis, H., \& Davidson, J. (1998). Attributional and affective responses of impostors to academic success and failure outcomes. Personality and Individual Differences, 25(2), 381-396. Doi: https://doi.org/10.1016/S0191-8869(98)00065-8

Thompson, T., Foreman, P., \& Martin, F. (2000). Impostor fears and perfectionistic concern over mistakes. Personality and Individual Differences, 29(4), 629-647.

Want, J., \& Kleitman, S. (2006). Imposter phenomenon and self-handicapping: Links with parenting styles and self-confidence. Personality and Individual Differences, 40(5), 961-971. Doi: https://doi. org/10.1016/j.paid.2005.10.005

Weiner, B. (1972). Attribution Theory, Achievement Motivation and The Educational Process. Review of Educational Research, 42(2), 203-215. 